\title{
SEMIPRIMITIVITY OF GROUP ALGEBRAS OF INFINITE SIMPLE GROUPS OF LIE TYPE
}

\author{
D. S. PASSMAN
}

(Communicated by Lance W. Small)

\begin{abstract}
Let $G$ be a simple group of Lie type over an infinite locally finite field $F$. For any field $K$, we prove that the group algebra $K[G]$ is semiprimitive. The argument here is a mixture of combinatorial and topological methods. Combined with earlier results, it now follows that any group algebra of an infinite locally finite simple group is semiprimitive. Furthermore, if the group is countably infinite, then the group algebra is primitive. In particular, if $G$ is a simple group of Lie type over the field $F$, then $K[G]$ is a primitive ring.
\end{abstract}

Let $G$ be a simple group of Lie type over an infinite locally finite field $F$ of characteristic $q$. Say $G \subseteq M_{n}(F)$, and use $\phi(x)$ to denote the characteristic polynomial of $x \in G$ in the variable $\zeta$. Let $U(G)$ denote the set of unipotent elements of $G$ so that $U(G)=G_{q}$ is the set of $q$-elements of $G$.

Now let $K$ be a field of characteristic $p>0$, and let $K^{t}[G]$ be a twisted group algebra of $G$ over $K$. We do not assume that $p$ and $q$ are distinct. Let $G_{p, q}$ denote the set of $(p, q)$-elements of $G$. Our goal is to prove that $K^{t}[G]$ is semiprimitive. Suppose, by way of contradiction, that this is not the case, and let

$$
0 \neq \alpha=1+\sum_{1}^{s} k_{i} \bar{x}_{i} \in J K^{t}[G]
$$

with each $1 \neq x_{i} \in G$.

Lemma 1. There exists a constant $c=c(p, q)$ such that $\left|q^{r}-1\right|_{p} \leq c r$ for all $r>0$.

Proof. This is trivial if $p=q$. Suppose $p \neq q$, and choose $e$ minimal with $p \mid\left(q^{e}-1\right)$ and $4 \mid\left(q^{e}-1\right)$ if $p=2$. Then, as is well known,

$$
\left|q^{r}-1\right|_{p} \leq\left|q^{e r}-1\right|_{p}=\left|q^{e}-1\right|_{p}|r|_{p} \leq\left(q^{e}-1\right) r,
$$

so the result follows with $c=q^{e}-1$.

The next lemma isolates a small part of the unipotent structure of $G$. See $[\mathrm{C}]$ or $[\mathrm{St}]$ for the information we require.

Received by the editors July 29, 1992 and, in revised form, September 29, 1992.

1991 Mathematics Subject Classification. Primary 16S34, 20E32, 20F50. 
Lemma 2. There exists a constant $l \geq 1$, matrices $v_{a, b} \in M_{n}(F)$ for $1 \leq$ $a, b \leq l$, and a field automorphism $\theta$ of $F$ such that:

(i) $u_{\lambda}=1+\sum_{a, b} \lambda^{a+\theta b} v_{a, b} \in U(G)$ for all $\lambda \in F$. Furthermore, $u_{\lambda}$ is not identically equal to 1 .

(ii) The restriction of the action of $\theta$ to any $\mathrm{GF}\left(q^{r}\right) \subseteq F$ is given by $\theta: \gamma \rightarrow$ $\gamma^{m(r)}$, where $1 \leq m(r) \leq q^{(r+1) / 2}$.

Proof. Let $\theta$ be the field automorphism used to construct $G$. If $\theta$ has order $\leq 2$, then $m(r)=1$ or $r$ is even and $m(r)=q^{r / 2}$. Otherwise, $G$ is a Suzuki or Ree group, $q=2$ or $3, r$ is odd, and $m(r)=q^{(r+1) / 2}$. Finally, $u_{\lambda}$ and the matrices $v_{a, b}$ are easily seen to exist by considering a nontrivial root subgroup of $G$ for a maximal root.

We now proceed to the combinatorial part of the proof using the above notation.

Lemma 3. Let $w \in G_{p, q}$ satisfy $\phi\left(u_{\lambda} w\right)=\phi(w)$ for all $\lambda \in F$. Then there exists $1 \leq i \leq s$ such that $w x_{i} \in G_{p, q}$ and $\phi\left(u_{\lambda} w x_{i}\right)=\phi\left(w x_{i}\right)$ for all $\lambda \in F$. Proof. Choose $\operatorname{GF}\left(q^{r}\right) \subseteq F$ so that $w$, all $v_{a, b}$, and all $x_{i}$ are contained in $M_{n}\left(q^{r}\right)$. There are of course infinitely many such $r$; note that the constants used below are all independent of $r$ unless otherwise indicated. Note also that $\phi\left(u_{\lambda} w\right)=\phi(w)$ implies that $u_{\lambda} w \in G_{p, q}$ for all $\lambda \in \operatorname{GF}\left(q^{r}\right)$.

Let $x$ be any $(p, q)$-element of $G$, and use the notation of [P]. Then $\mathbb{C}_{G}^{t}(\bar{x})=\mathbb{C}_{G}(x)$ since $K$ has characteristic $p$ and the Schur multiplier of $G$ is a finite group of order prime to $q$ (see [G, Theorem 4.235] or [St]). Furthermore, if $x \in \mathrm{GL}_{n}\left(q^{r}\right)$ and $\varepsilon$ is an eigenvalue of $x$, then $\varepsilon$ is a $p$-element in $\mathrm{GF}\left(q^{n ! r}\right)$. Hence, by Lemma 1 , there are at most $c^{\prime} r$ choices for $\varepsilon$, where $c^{\prime}$ is a constant independent of $r$. In particular, if $c=\left(c^{\prime}\right)^{n}$, then there are at most $c r^{n}$ choices for the characteristic polynomial $\phi(x)$. Say these possibilities are $\phi_{1}, \phi_{2}, \ldots, \phi_{d}$, where $d=d(r) \leq c r^{n}$.

For each $1 \leq i \leq s$ and $1 \leq j \leq d(r)$, let

$$
F_{i, j}=\left\{\lambda \in \mathrm{GF}\left(q^{r}\right) \mid \phi\left(u_{\lambda} w x_{i}\right)=\phi_{j}\right\} .
$$

We claim that $\bigcup_{i, j} F_{i, j}=\operatorname{GF}\left(q^{r}\right)$. To this end, let $\lambda \in \operatorname{GF}\left(q^{r}\right)$. Since $u_{\lambda} w$ and all its powers are $(p, q)$-elements, $[\mathrm{P}$, Lemma 6.2] and the preceding comments on $\mathbb{C}_{G}^{t}$ imply that there exist $k, i$ with

$$
\left(u_{\lambda} w x_{i}\right)^{p^{k}} \sim\left(u_{\lambda} w\right)^{p^{k}}
$$

where $\sim$ indicates that the elements are $G$-conjugate. In particular, $u_{\lambda} w x_{i}$ is a $(p, q)$-element in $\mathrm{GL}_{n}\left(q^{r}\right)$, so $\phi\left(u_{\lambda} w x_{i}\right)=\phi_{j}$ for some $j$ and $\lambda$ is contained in $F_{i, j}$.

Suppose that, for all $i, j$, we have

$$
\left|F_{i, j}\right| \leq n l q^{(r+1) / 2} \text {. }
$$

Since $\operatorname{GF}\left(q^{r}\right)=\bigcup_{i, j} F_{i, j}$, this yields

$$
q^{r}=\left|\mathrm{GF}\left(q^{r}\right)\right| \leq s d(r) n l q^{(r+1) / 2} \leq s c r^{n} n l q^{(r+1) / 2},
$$

so $q^{(r-1) / 2} \leq s c n l r^{n}$, an equation which certainly cannot hold for all such $r$. Thus, when $r$ is taken to be sufficiently large, there exist subscripts $i$ and $j$ with

$$
\left|F_{i, j}\right|>n l q^{(r+1) / 2}
$$


Now notice that, by Lemma 2, the coefficients of $\phi\left(u_{\lambda} w x_{i}\right)$ are all polynomials in $\lambda$ of degree $\leq n l q^{(r+1) / 2}$. In particular, if one of these polynomials is not identically constant, then the number of $\lambda \in \mathrm{GF}\left(q^{r}\right)$ with $\phi\left(u_{\lambda} w x_{i}\right)=\phi_{j}$ is bounded by this $\lambda$-degree, a contradiction. Thus these polynomials must all be identically constant, and therefore, for all $\lambda \in F$, we have

$$
\phi\left(u_{\lambda} w x_{i}\right)=\phi_{j}=\phi\left(u_{0} w x_{i}\right)=\phi\left(w x_{i}\right) .
$$

Since $\phi\left(w x_{i}\right)=\phi_{j}$, it follows that $w x_{i} \in G_{p, q}$, and the lemma is proved.

Now let $G \subseteq \mathrm{GL}_{n}(F)$ be endowed with the Zariski topology. As will be apparent, the remainder of the argument is topological in nature. Define

$$
W=\left\{w \in G_{p, q} \mid \phi\left(g^{-1} u_{\lambda} g w\right)=\phi(w) \text { for all } g \in G, \lambda \in F\right\},
$$

and set

$$
W^{\prime}=\left\{w \in G \mid \phi\left(g^{-1} u_{\lambda} g w\right)=\phi(w) \text { for all } g \in G, \lambda \in F\right\} .
$$

Notice that $W \subseteq W^{\prime}$ and $W^{\prime}$ is Zariski closed in $G$. Thus, if $\bar{W}$ denotes the closure of $W$ in $G$, then $W \subseteq \bar{W} \subseteq W^{\prime}$. Furthermore, $1 \in W \subseteq \bar{W}$ since $u_{\lambda}$ is unipotent. Recall that a topological space is irreducible if it is not the union of two closed proper subspaces.

Lemma 4. If $\bar{w} \in \bar{W}$, then $\bar{w} x_{i} \in \bar{W}$ for some $1 \leq i \leq s$.

Proof. First let $w \in W$ and, for each $i$ with $w x_{i} \in G_{p, q}$, let

$$
B_{i}=\left\{g \in G \mid \phi\left(g^{-1} u_{\lambda} g w x_{i}\right)=\phi\left(w x_{i}\right) \text { for all } \lambda \in F\right\} .
$$

Since $w$ is fixed, it is clear that each $B_{i}$ is a Zariski closed subset of $G$. If $g \in G$, then $g^{-1} u_{\lambda} g=1+\sum_{a, b} \lambda^{a+\theta b} g^{-1} v_{a, b} g \in U(G)$, and therefore Lemma 3 , with $u_{\lambda}$ replaced by $g^{-1} u_{\lambda} g$, implies that there exists $i$ with $w x_{i} \in G_{p, q}$ and

$$
\phi\left(g^{-1} u_{\lambda} g w x_{i}\right)=\phi\left(w x_{i}\right)
$$

for all $\lambda \in F$. In other words, $g \in B_{i}$, and we have shown that $G=\bigcup_{1}^{s} B_{i}$. Furthermore, $G$ is connected by [W, Lemma 5.2], so we conclude from [W, Lemma 14.3] that $G$ is irreducible. Thus $G=B_{j}$ for some $j$, and hence $w x_{j} \in W$.

Now let $\bar{w} \in \bar{W}$, and suppose, by way of contradiction, that no $i$ exists with $\bar{w} x_{i} \in \bar{W}$. Then, for each $i$, there exists an open subset $\mathscr{O}_{i}$ of $G$ with $\bar{w} x_{i} \in \mathscr{O}_{i}$ and $\mathscr{O}_{i} \cap W=\varnothing$. Now each $\mathscr{O}_{i} x_{i}^{-1}$ is open and contains $\bar{w}$, so $\mathscr{O}=\bigcap_{1}^{s} \mathscr{O}_{i} x_{i}^{-1}$ is an open neighborhood of $\bar{w} \in \bar{W}$. Therefore, there exists $w^{\prime} \in \mathscr{O} \cap W$. Finally, by the result of the previous paragraph, $w^{\prime} x_{k} \in W$ for some $k$ and hence $w^{\prime} x_{k} \in \mathscr{O}_{k} \cap W$, a contradiction

Since the closed subsets of $\bar{W}$ satisfy the descending chain condition, it follows from the proof of [W, Lemma 14.3] that $\bar{W}=C_{1} \cup C_{2} \cup \cdots \cup C_{t}$ is uniquely a finite irredundant union of closed irreducible subspaces $C_{i}$. We call these $C_{i}$ the irreducible components of $\bar{W}$.

Lemma 5. $\bar{W}$ and each $C_{j}$ are stable under the conjugation action of $G$. Furthermore, for each irreducible component of $\bar{W}$, there exist $x_{i}$ and an irreducible component $C^{\prime}$ with $C x_{i} \subseteq C^{\prime}$.

Proof. If $w \in W$ and $y \in G$, then

$$
\phi\left(y^{-1} w y\right)=\phi(w)=\phi\left(y g^{-1} u_{\lambda} g y^{-1} w\right)=\phi\left(g^{-1} u_{\lambda} g y^{-1} w y\right)
$$


for all $\lambda \in F$ and $g \in G$. Since $y^{-1} w y \in G_{p, q}$, it follows that $y^{-1} w y \in W$. In other words, $W$ is stable under $G$-conjugation, and hence so is $\bar{W}$. This implies that $G$ permutes the finitely many irreducible components $C_{j}$ and, since $G$ has no proper subgroup of finite index, the first part is proved.

Next, notice that

$$
\bar{W}_{i}=\left\{\bar{w} \in \bar{W} \mid \bar{w} x_{i} \in \bar{W}\right\}=\bar{W} \cap \bar{W} x_{i}^{-1}
$$

is a closed subset of $\bar{W}$ and that $\bar{W}=\bigcup_{1}^{s} \bar{W}_{i}$ by the previous lemma. Thus $C=\bigcup_{1}^{s}\left(\bar{W}_{i} \cap C\right)$ and, since $C$ is irreducible, it follows that $\bar{W}_{i} \supseteq C$ for some $i$. In other words, $C x_{i} \subseteq \bar{W}$ and, since $C x_{i}$ is irreducible and $C x_{i} \subseteq \bigcup_{1}^{t} C_{j}$, we have $C x_{i} \subseteq C^{\prime}$ for some appropriate $C^{\prime}$.

We can now complete the proof of

Theorem 6. If $G$ is a simple group of Lie type over an infinite locally finite field $F$, then any twisted group algebra $K^{t}[G]$ is semiprimitive.

Proof. If $K$ has characteristic 0 , then $K^{t}[G]$ is semiprimitive for any locally finite group $G$. Thus it suffices to assume that char $K=p>0$. We continue with the preceding notation. In addition, let $\mathscr{X}$ denote the set $\left\{x_{i}, x_{2}, \ldots, x_{s}\right\}$, and write $\mathscr{C}=\left\{C_{1}, C_{2}, \ldots, C_{t}\right\}$.

Let $C(1)$ be any irreducible component of $\bar{W}$. Then, by applying the previous lemma repeatedly, we can construct a sequence $C(1), C(2), C(3), \ldots$ of elements of $\mathscr{C}$ and a sequence $y_{1}, y_{2}, y_{3}, \ldots$ of elements of $\mathscr{X}$ such that $C(k) y_{k} \subseteq C(k+1)$ for all $k \geq 1$. Since $|\mathscr{C}|=t<\infty$, it follows that $C=C(i)=C(j)$ for some $i<j$. Set $D=C(i+1)$, and note that $C y_{i} \subseteq D$ and $D z \subseteq C$ where $z=y_{i+1} \cdots y_{j-1} \in G$. But $C$ and $D$ are $G$-stable, so $C g^{-1} y_{i} g \subseteq D$, and hence $C g^{-1} y_{i} g z \subseteq C$ for all $g \in G$. In particular, since $y_{i} \neq 1$, it follows that $y_{i}$ is not central, and therefore we can find $1 \neq h \in G$ with $C h \subseteq C$.

Let $H=\{g \in G \mid C g \subseteq C\}$. Then $H$ is surely closed under multiplication, so $H$ is a subgroup of the periodic group $G$. Furthermore, since $C$ is $G$-stable, it follows that $H \triangleleft G$. Thus, since $H \neq 1$ by the above, we conclude that $H=G$ and therefore that $C \supseteq C G=G$. In other words, $C=G$, so $\bar{W}=G$, and hence $W^{\prime}=G$.

Finally, write $U=U(G)$, and let $L=\{g \in G \mid g U \subseteq U\}$. Again it is clear that $L \triangleleft G$. Furthermore, as we will see, $u_{\lambda} \in L$ for all $\lambda \in F$. Indeed, if $w \in U$, then $w \in G=W^{\prime}$, so $\phi\left(u_{\lambda} w\right)=\phi(w)$ for all $\lambda \in F$. Since $w$ is unipotent, this implies that $u_{\lambda} w$ is also unipotent, and hence $u_{\lambda} w \in U$, as required. Furthermore, by Lemma $2, u_{\lambda}$ is not identically equal to 1 , so it follows that $L \neq 1$ and therefore that $L=G$. But then $G U \subseteq U$, so $G=U$ is unipotent, a contradiction.

A number of corollaries now follow quite quickly. To start with, if $G$ is an infinite locally finite simple group which is also a linear group, then by [HS, Theorem B] or [T, Theorem 2] we know that $G$ must be a simple group of Lie type over an infinite locally finite field. Thus the preceding result, along with [PZ, Theorem 1.1] and the remark at the end of that paper, yields

Corollary 7. Let $G$ be an infinite locally finite simple group. Then any twisted group algebra $K^{t}[G]$ is semiprimitive.

Next, we conclude from [FS, Theorem 2.2] and the above that 
Corollary 8. Let $G$ be a countably infinite locally finite simple group. Then any twisted group algebra $K^{t}[G]$ is primitive. In particular, this applies to the simple groups of Lie type over infinite locally finite fields.

Finally, by Theorem 6 and the argument of [P, Theorem 6.1], we obtain

Corollary 9. Let $K$ be a field of characteristic $p>0$, and let $G$ be a locally finite group having a finite subnormal series

$$
1=G_{0} \triangleleft G_{1} \triangleleft \cdots \triangleleft G_{n}=G
$$

with each quotient $G_{i} / G_{i-1}$ either (i) a p'-group, or (ii) a finite simple group, or (iii) a simple group of Lie type over an infinite locally finite field. Then $J K^{t}[G]$ is nilpotent, so

$$
J K^{t}[G]=J K^{t}\left[\Delta^{p}(G)\right] K^{t}[G]
$$

with $\Delta^{p}(G)$ finite. Furthermore, $K^{t}[G]$ is semiprimitive if $G$ has no finite normal subgroup of order divisible by $p$.

We remark in closing that $[\mathrm{Z}]$ contains a direct proof that the ordinary group algebra $K[G]$ is primitive if $G$ belongs to one of the classical families $\mathrm{PSL}_{n}, \mathrm{PSp}_{n}, \mathrm{P}_{n}$, or $\mathrm{PSU}_{n}$, all with $p \neq q$. The argument uses known bounds on the composition lengths of certain permutation modules associated with the finite simple groups in these families.

\section{REFERENCES}

[C] R. W. Carter, Simple groups of Lie type, Wiley-Interscience, London, 1972.

[FS] J. W. Fisher and R. L. Snider, Prime von Neumann regular rings and primitive group algebras, Proc. Amer. Math. Soc. 44 (1974), 244-250.

[G] D. Gorenstein, Finite simple groups, Plenum Press, New York, 1982.

[HS] B. Hartley and G. Shute, Monomorphisms and direct limits of finite groups of Lie type, Quart. J. Math. Oxford (2) 35 (1984), 49-71.

[P] D. S. Passman, Semiprimitivity of group algebras of locally finite groups (to appear).

[PZ] D. S. Passman and A. E. Zalesskii, Semiprimitivity of group algebras of locally finite simple groups, Proc. London Math. Soc. (3) (to appear).

[St] R. Steinberg, Lectures on Chevalley groups, Yale Univ., 1967.

[T] S. Thomas, The classification of simple linear groups, Arch. Math. (Basel) 41 (1983), 103116.

[W] B. A. F. Wehrfritz, Infinite linear groups, Springer, Berlin, 1973.

[Z] A. E. Zalesskii, Semisimplicity of the group rings of classical locally finite simple groups, private communication, 1992.

Department of Mathematics, University of Wisconsin-Madison, Madison, Wisconsin 53706

E-mail address: passman@math.wisc.edu 